\title{
On simplicity and emergence
}

\author{
Andrew Perfors ${ }^{1}$
}

Published online: 24 January 2017

(C) Psychonomic Society, Inc. 2016

\begin{abstract}
Mark Johnson's paper centres around a provocative and sensible point: the simplicity of a change in evolutionary terms does not necessarily map straightforwardly onto the simplicity of that change within aformal system. This commentary discusses these implications in more depth, making the point that it isimportant to consider the level at which an operation occurs in order to correctly evaluate how complex it is.
\end{abstract}

Keywords Computational modeling · High order cognition

Mark Johnson's paper (2016) centers around a provocative and sensible point: The simplicity of a change in evolutionary terms does not necessarily map straightforwardly onto the simplicity of that change within a formal system. He discusses the implications of this point for the Minimalist Program, since one of its fundamental justifications for the likely evolutionary emergence of Merge is its computational simplicity.

It is incontestable that Merge is, computationally, an extremely simple operation - one with dramatic effects on the representational capacity of the resulting system. For this and other reasons well covered by Berwick and Chomsky (2016), it seems at least plausible that Merge is an important part of humanity's linguistic repertoire (a repertoire that is not shared with nonhuman animals).

Moreover, many scholars do agree that language may have emerged fairly abruptly, evolutionarily speaking. These two points, taken together, are often used to suggest that the

Andrew Perfors

perfors@gmail.com

1 School of Psychology, University of Adelaide, Adelaide, SA, Australia evolutionary change that occurred was the existence of Merge. The necessary additional premise making this argument plausible is that Merge resulted from a relatively simple genetic change or mutation, otherwise how could it have emerged in the first place? Such simplicity is especially a consideration given that Merge is so computationally simple and so distinct to humans: The Minimalist Program posits no such thing as a half-Merge or an operation that could have served as an evolutionary bridge. As a result, the argument goes, because Merge is a single factor that must have emerged spontaneously, it is highly probable that it is fairly simply represented in the genome.

This is where Johnson's point is relevant. Just because Merge is simple computationally does not mean that it is simple on the genomic, implementational, or hardware level. Johnson's analogy with the wiring diagram of a calculator is apt. If Merge is not simple on an implementation level, that makes the argument that it was the evolutionary change underlying the emergence of language much more difficult to believe.

But why should simplicity matter anyway? It is true that simplicity is a guiding principle in many areas of science: The ever-more-complex epicycles needed to account for the motion of the planets was one of the main reasons to support the heliocentric theory. But why? Besides arguments from aesthetics - simple theories are more beautiful — the main reason to prefer a simpler theory is that it is more plausible: There are fewer "choice points" at which something else could have happened.

This is where the level on which theories are simple matters: The level of the "choice points" must map onto the level at which the claim is being made. If one is speaking of evolutionary theory, it matters that the simplicity be on that level: Here the "choice points" are mutations and genetic factors, since that is what was hypothesized to have changed. The 
more complicated the change at that level - say, multiple interlocking mutations or complex interactions that must take place over embryonic development - the less plausible it was for that change to occur.

I am not saying that simplicity on the computational level is not interesting or important. Indeed, it is an important factor in model selection for a reason: Because the more computationally complex a model or a theory is, the more difficult it is, plausibly, to represent or learn. For those reasons the simplicity of Merge is a theoretical asset when evaluating its cognitive plausibility.
However, its computational simplicity does not increase the plausibility that it arose over the course of evolution.

\section{References}

Berwick, R., \& Chomsky, N. (2016). Why only us: Language and evolution. Cambridge: MIT Press.

Johnson, M. (2016). Marr's levels and the Minimalist program. Psychonomic Bulletin \& Review. doi:10.3758/s13423-016-1062-1 